\title{
Ethnologies
}

\section{Le rôle du patrimoine dans la construction identitaire et géopolitique de la Corée du Sud}

\section{Jung Sook Bae}

Volume 39, numéro 1, 2017

Géopolitique, conflits et patrimoine

Geopolitics, conflicts and heritage

URI : https://id.erudit.org/iderudit/1051058ar

DOI : https://doi.org/10.7202/1051058ar

Aller au sommaire du numéro

Éditeur(s)

Association Canadienne d'Ethnologie et de Folklore

ISSN

1481-5974 (imprimé)

1708-0401 (numérique)

Découvrir la revue

Citer cet article

Bae, J. (2017). Le rôle du patrimoine dans la construction identitaire et géopolitique de la Corée du Sud. Ethnologies, 39(1), 175-187.

https://doi.org/10.7202/1051058ar
Résumé de l'article

$\mathrm{Au}$ cours de son histoire, après une période de destruction systématique, la Corée du Sud a su procéder à une réappropriation patrimoniale et culturelle progressive et adaptée à son contexte socioculturel et économique. Les planifications à long terme successives du développement ont permis aux Coréens d'inventer des concepts originaux et de mettre de nouvelles technologies au service de la conservation, de la protection et de la valorisation du patrimoine culturel. La combinaison de la culture traditionnelle et des nouvelles technologies est bénéfique au niveau artistique, avec des résultats parfois très étonnants pour ceux qui ne sont pas familiers avec cet environnement socioculturel. La tendance à la globalisation et à

l'internationalisation est présente, mais un certain conservatisme nationaliste reste un frein récurrent. Cette étude présente une vue globale du processus politique de réappropriation du patrimoine culturel, depuis l'indépendance de la Corée jusqu'à nos jours. La Corée du Sud a su instaurer un cadre institutionnel à différents niveaux d'organisation pour protéger, voire développer, ses acquis patrimoniaux. Elle propose les aspects les plus originaux et les plus efficients de son programme culturel (dont la notion de « bien culturel immatériel ») à la communauté internationale. Un exemple significatif illustre l'évolution historique du bien culturel immatériel le plus précieux des Corées : le système d'écriture hangeul.
Ce document est protégé par la loi sur le droit d'auteur. L'utilisation des services d'Érudit (y compris la reproduction) est assujettie à sa politique d'utilisation que vous pouvez consulter en ligne.

https://apropos.erudit.org/fr/usagers/politique-dutilisation/ 


\title{
LE RÔLE DU PATRIMOINE DANS LA CONSTRUCTION IDENTITAIRE ET GÉOPOlITIQUe DE LA CORÉE DU Sud
}

\author{
Jung Sook Bae \\ Université de Bourgogne-Franche-Comté (UTBM)
}

\section{Introduction}

Les patrimoines culturels en Corée du Sud sont protégés par des lois de protection du patrimoine culturel, la première d'entre elles étant entrée en vigueur en 1962. Elles permettent d'entreprendre des actions concrètes pour conserver et protéger le patrimoine culturel, dans lequel on distingue quatre types : le patrimoine matériel, le patrimoine immatériel, le patrimoine historique et l'héritage culturel de la tradition. À cette classification thématique se superpose un classement par niveau d'importance : patrimoine national, patrimoine régional, archives culturelles, patrimoine classé et patrimoine "non classé ». La Corée du Sud a su instaurer un cadre institutionnel assez tôt, dès les années 1960, car il s'agissait pour elle de consolider son identité blessée et niée. Le patrimoine fut un facteur de résilience et de cohésion sociale après le viol culturel japonais, mais aussi un vecteur d'affirmation identitaire et géopolitique face à la Corée du Nord. L'importance conférée à l'enjeu patrimonial est restée constante sous tous les régimes politiques qu'elle a connus, jusqu'à aujourd'hui avec la création d'une université dédiée au patrimoine. Le sens même donné à l'enjeu patrimonial a évolué pour devenir une source de bien-être, d'harmonie et de réconciliation, y compris avec la Corée du Nord. La culture a été un élément précurseur du processus de rapprochement politique des deux Corées.

\section{Le patrimoine culturel coréen, un enjeu identitaire et géopolitique après l'occupation japonaise}

En 1948, le premier gouvernement coréen fut fondé après trois ans de tutelle des Nations Unies, tutelle mise en œuvre par l'armée américaine après la libération de la colonisation japonaise (1910-1945). Ensuite, la 
guerre de Corée (1950-1953) laissa le pays complétement dévasté. Cette période fut très difficile pour la politique du patrimoine culturel. En effet, les conflits sociétaux étaient omniprésents, ainsi qu'une confusion culturelle provoquée par la longue période durant laquelle le pays avait été soumis à la politique culturelle japonaise qui visait à l'assimiler intégralement à l'identité japonaise (pour alimenter la machine de guerre nippone), ponctuée par une relative période de tolérance vis-à-vis d'un développement culturel purement coréen. L'orientation politique fondamentale du premier gouvernement était l'anticommunisme et la réunification de la péninsule coréenne pour apaiser l'instabilité politique du Sud, et pour ce faire, le développement économique était le premier objectif. Dans ce cadre, la politique culturelle devait viser six objectifs :

conservation et protection du patrimoine culturel matériel ;

promotion de la culture nationale de la Corée ;

réforme des établissements culturels créés durant la colonisation japonaise et rétablissement de la structure institutionnelle de l'art et de la culture coréenne ;

élimination de l'influence de la culture communiste et gauchiste ;

initiation d'une approche harmonieuse entre la culture traditionnelle et la culture libérale démocratique ;

initiation à l'activité culturelle en associant l'enseignement et la culture.

(Korea Culture and Arts Agency, Korea's Cultural Policy 1992)

Dès la Première République sont créées les premières institutions dédiées à la culture. Le bilan des initiatives prises dans les années 1950 est loin d'être négligeable. Voici quelques créations emblématiques : Institut national de la Musique traditionnelle, directement rattaché au ministère de l'Éducation ; Institut d'Art ; Institut académique ; politique de soutien aux artistes ${ }^{1}$; établissement de formation des musiciens traditionnels (devenu aujourd'hui le lycée national de la musique traditionnelle) ; Théâtre national ; protocole de la Loi sur la protection du patrimoine. Un événement symbolise cette politique : en 1958, le concours national d'art traditionnel devient une grande fête nationale pour célébrer la $10^{\mathrm{e}}$ année de sa création par le gouvernement ; ce concours a contribué cette année-là

1. L'Université nationale de Séoul a créé sa faculté des arts en 1948 et sa faculté de musique traditionnelle coréenne en 1959. 
à la découverte de la culture immatérielle par diverses activités culturelles et par le talent des artistes en lice (An 2004).

Pourtant, les difficultés semblaient insurmontables. Définir des objectifs ambitieux et cohérents dans un environnement d'instabilité politique et idéologique tenait déjà du miracle, mais leur trouver un financement alors que les besoins matériels étaient criants, cela démontre une volonté forte de ne pas sacrifier la Culture à l'Économie. Les artistes se préoccupaient davantage de survie et de combat idéologique que de création culturelle, même si de nombreux groupes et associations culturelles avaient éclos. La coexistence de la propagande coloniale et de l'idéologie conservatrice nationale étaient sources de tensions et la Corée vivait une situation paradoxale avec l'entrée massive de la culture occidentale libérale, a priori favorable au mouvement de la démocratisation, alors que le régime en place justifiait son autoritarisme par la menace du Nord. Car la Troisième République de Corée est née d'un coup d'État militaire (le 16 mai 1961). Le gouvernement de Park Chung-hee visait d'abord la modernisation du pays et le développement économique. Il promulgua bien une loi d'administration de la politique culturelle, mais celle-ci se focalisait sur le contrôle nationaliste et anticommuniste du processus administratif. Le gouvernement militaire a promulgué de nombreuses lois s'appliquant à l'activité culturelle et à la gestion des biens culturels. Elles concernent les spectacles (1961) ; l'enregistrement de l'édition et de l'impression (1961) ; la protection du patrimoine culturel (1962) ; la gestion des biens des temples bouddhiques de l'école confucéenne (1962); l'organisation de l'activité culturelle régionale (1965) ; l'activité cinématographique (1966); et l'enregistrement de la musique (1967).

Un élément intéressant est à noter : à l'occasion de la création du premier établissement de gestion du patrimoine, la notion de " patrimoine culturel » apparaît officiellement pour la première fois. Et c'est en 1962 que la Loi sur la protection du patrimoine a introduit explicitement la notion de «patrimoine immatériel ».

\section{Patrimoine immatériel, " trésor national " et " trésor humain vivant "}

La Corée du Sud fait figure de pionnière et de référence internationale dans la reconnaissance du patrimoine immatériel dont on parle tant aujourd'hui et qui est devenu un axe majeur de la politique de l'UNESCO. Elle l'a institué par la création des « trésors humains vivants » qui fut un facteur important de la revitalisation culturelle de la Corée du Sud et de 
sa réputation. Un « trésor humain vivant » est une notion peu connue hors de la Corée. Il désigne un art ou un artisan reconnu dans une activité traditionnelle, qui s'engage - en tant que collectivité ou individuellement - à transmettre son savoir à un ou plusieurs apprentis. Cette activité ou cet artisan bénéficient, du fait du décret de nomination, d'une publicité gratuite et par ailleurs d'une subvention non négligeable. La réflexion ayant mené à la création de cette institution est très bien rendue par cette citation du journaliste Soul Ho-jeong.

Au temps où le patrimoine culturel ne se concevait que par les palais royaux et les pagodes en pierre, il se trouvait des gens pour affirmer avec constance que les chamanes n'avaient pas moins de valeur que les biens culturels matériels. Leur engagement pour cette cause a permis de garder en vie nombre d'aspects du patrimoine culturel immatériel qu'une industrialisation effrénée menaçait de faire disparaître à jamais. (Soul Ho-jeong 2012:3)

Les quatre premières nominations nationales ont été proclamées en 1966. Elles sont prononcées chaque année et on en compte actuellement 250. Les nominations provinciales sont bien plus nombreuses, mais n'ont pas le même prestige. D'un avis unanime en Corée, le nombre de nominations nationales est insuffisant (et cela tient à des contraintes budgétaires). Trois exemples de « trésors humains vivants » illustrent très bien la variété de ces distinctions patrimoniales, mais aussi l'importance accordée au patrimoine immatériel dès les années 1960. Le premier est le chant « Pansori », dont les interprètes ont une technique vocale très particulière soutenue par un tambour qui produit aussi des interjections d'encouragement. Le deuxième exemple est celui d'un producteur d'alcool traditionnel. Mais c'est un autre exemple qui nous permettra d'illustrer la manière dont la Corée a abordé une distinction patrimoniale importante comme moyen de protéger son identité (le «trésor national ») : l'écriture coréenne hangeul.

Le hangeul est une écriture inventée après l'étude du système d'articulatoire et des caractéristiques phonétiques de l'oralité du coréen en 1443 par le roi Lee Sejong, et qui fut proclamé « Hunminjeongeum », ce qui signifie littéralement « les sons corrects/ appropriés pour l'instruction du peuple ». Ce Trésor national a été enregistré en tant que patrimoine mémoriel par l'UNESCO en octobre 1997. Cette écriture si originale est unique par son apport objectif au niveau culturel élevé des Coréens d'aujourd'hui, mais aussi par sa grande valeur symbolique. Jusqu'au milieu du $X V^{e}$ siècle, les érudits coréens écrivaient en chinois mandarin. S’ils condescendaient à s'exprimer en langue vernaculaire, ils n'utilisaient que 
les caractères chinois, soit pour leur sens, soit pour leur prononciation. Seuls les membres de la classe des lettrés, ayant énormément de temps disponible pour l'étude, pouvaient accéder à la littérature. Mais un très grand roi, Sejong (1397-1450), conscient de l'énorme gâchis lié à l'illettrisme, fit développer et diffuser un alphabet phonémique très simple, construit sur des bases rationnelles. Les conservateurs, dès cette époque, s'opposèrent à cette perte de leurs privilèges exclusifs. Après la disparition de Sejong, le hangeul fut souvent interdit et survécut difficilement comme "écriture des femmes ». Paradoxalement, l'occupation japonaise représenta une grande chance pour le développement du hangeul. Inconnu de l'occupant japonais, il devint une manifestation de résistance et une sorte de langage codé. Le hangeul s'est développé pendant une bonne décennie de tolérance, puis il fut interdit sous peine de sanctions graves pendant la période finale d'assimilation au Japon.

Après la séparation des deux Corées, le sort du hangeul diverge. Au Nord, la politique d'autonomie appelée " juche » supprima totalement l'usage des caractères chinois, coupant par-là l'accès aux textes anciens pour les jeunes générations. Au Sud, il était de bon ton d'utiliser une écriture mixte : le hangeul pour les mots purement coréens et les syllabes grammaticales; les caractères chinois pour la racine des mots venant de cette langue sino-coréenne. On trouve peu de publications en hangeul pur au début de la libération, malgré les efforts suivis des autorités pour limiter le nombre de caractères chinois utilisables (1800 caractères quand même). La tendance actuelle au Sud est à l'abandon progressif des caractères chinois. Aujourd'hui, on observe donc sur ce point une convergence spontanée Nord-Sud de l'utilisation de ce patrimoine culturel commun. Seules la simplicité et la rationalité du hangeul ont permis à la Corée d'atteindre un niveau d'analphabétisme de l'ordre de moins de $1 \%$, ce qui est aussi un prérequis du développement socioculturel et économique.

\section{Le patrimoine comme moyen politique d'affirmation d'une identité nationale autonome}

On s'aperçoit donc que la question patrimoniale sert le projet politique global de ce régime non démocratique et nationaliste car il lui permet de donner corps à un mouvement de restitution d'une identité violée par l'occupant japonais. Cependant, dix ans après, en 1972, est promulguée la Loi de la promotion de la culture et de l'art. On considère que la véritable politique de la culture commence à cette période, bien que la société subisse toujours les convulsions des conflits politiques et des vigoureuses 
manifestations pro-démocratiques. La politique culturelle est centrée sur l'accentuation de l'identité nationale en vue de soutenir la défense et le développement économique.

Le patrimoine culturel est donc regardé par le pouvoir politique comme un élément de prestige et de soft power, mais aussi de contrôle social. En témoigne le premier Plan quinquennal de promotion de la culture mis en œuvre en 1973. Il visait trois objectifs : établir la représentation « correcte » de l'histoire nationale ; élever le niveau de la culture du peuple en activant et en popularisant l'art et la culture ; rehausser le prestige national en promouvant les échanges internationaux avec la culture coréenne. C'est toujours dans cette perspective que furent créés en 1975 l'Institut de recherche sur le patrimoine et le Musée national de la Tradition. Mais l'impératif de réappropriation patrimoniale devait concerner tout le territoire. C'est pourquoi, en 1977, il incombait à chaque région de mettre en place un département de gestion du patrimoine culturel. Au niveau national on voit apparaître l'Institut national de la recherche sur le patrimoine culturel immatériel. Le deuxième Plan quinquennal de promotion de la culture, lancé en 1978, renforce cette dynamique : recherche du développement de la culture traditionnelle ; création de la nouvelle culture coréenne fondée sur la tradition ; répartition équilibrée des bénéfices de la culture à tout le peuple ; contribution de la culture coréenne à la culture mondiale. Cependant, malgré ce tropisme nationaliste, les échanges internationaux se multiplient, ce qui a amélioré la visibilité mondiale de la culture coréenne. Le constat des différences a permis une renaissance de l'art traditionnel de la Corée et la valorisation de son patrimoine. Des moyens financiers importants ont été mobilisés. Les entreprises ont participé à cet effort, en contrepartie de quoi elles ont pu bénéficier de réductions d'impôts.

La Corée vit alors une situation que l'on pourrait qualifier de bipolaire : d'un côté, un processus d'intensification de la modernisation (économique et technique) du pays, de l'autre côté, une volonté de redécouverte des traditions qui se traduit par un surinvestissement dans l'enjeu patrimonial. Les deux dimensions ne sont pas contradictoires si on les replace dans un contexte politique marqué à la fois par l'anticommunisme et par le nationalisme, et qui s'inscrit dans une revendication forte de soigner les séquelles de l'occupation nippone. C'est par l'économie et la culture que la Corée du Sud entend dépasser la Corée du Nord et montrer au monde que son modèle est le plus performant. Le régime militaire du général Chun Doo-hwan (devenu le nouveau président de la République en 
septembre 1980 après avoir renversé son prédécesseur Park) correspond au «miracle économique » sud-coréen, caractérisé par des taux de croissance économique élevés. Malgré la répression politique continue, la tenue des Jeux olympiques à Séoul en 1988 fut un succès international et mit un terme à l'image misérabiliste de la Corée du Sud. Face à l'ampleur des mouvements de contestation du régime qui revendiquaient la démocratie, le général Chun Doo-hwan accepte que l'élection présidentielle se fasse au suffrage universel direct en décembre 1987. Le candidat qu'il soutient, le général Roh Tae-woo est élu. L'ancien bras droit de Chun Doo-hwan rompt toutefois avec la politique de son prédécesseur. Le mandat du président Roh Tae-woo (1988-1993) a été marqué par l'entrée conjointe des deux Corées à l'ONU en 1991. Nous vivons alors un changement planétaire imprévu : l'effondrement de l'URSS et la fin de la guerre froide. Un autre élément, culturel celui-là, pourrait être pris en compte. En effet, dans un monde imprégné de culture confucéenne, où le "savoir » est le bien le plus important, au moins en théorie, donner de l'importance à la culture nationale, n'est-ce pas aussi un moyen de conserver le pouvoir politique?

Les années 1980 se traduisent par un processus d'élargissement de la notion d'héritage culturel et d'amélioration de la conservation du patrimoine. La politique culturelle est toujours liée à l'affirmation d'une identité nationale autonome, mais s'y ajoutent trois objectifs : mettre les biens culturels à la disposition du public ; promouvoir la professionnalisation de la culture ; rehausser son niveau artistique. La politique de la culture est devenue la principale préoccupation de l'État, mais il ne s'agit pas que d'une question de prestige : un travail très concret de re-patrimonialisation a donné des résultats remarquables. Dans les années 1980, 136 travaux de restauration de biens culturels ont été réalisés. Les travaux de conservation de l'héritage culturel, la recherche scientifique qui lui est liée, la construction des villages folkloriques traditionnels sont activement poursuivis. La conservation et la formation des patrimoines immatériels sont poussées avec des soutiens gouvernementaux.

\section{Le nouveau paradigme culturel de l'après-guerre froide : le patrimoine comme facteur de "reliance " et d'harmonie}

À partir des années 1990, le contexte politique et géopolitique change considérablement, même si la Corée du Nord représente une persistance résiduelle et intempestive de la guerre froide. Ce changement va-t-il avoir des effets sur la politique patrimoniale et culturelle de la Corée du Sud? 
Le ministère de la Culture est né en 1990, trois ans après la création de la Sixième République. Le Plan décennal de développement de la culture est mis en route la même année. Il s'agit d'un moment important de promotion de la culture dans l'histoire coréenne. Le slogan «Culture pour tous les peuples » est affiché dès la naissance de ce ministère et la nouvelle stratégie de la culture mise en œuvre selon le principe du « culturalisme » (Rozin 2006). Le culturalisme est une doctrine anthropologique qui considère que l'influence du milieu culturel - des formes acquises de comportement sur la formation des individus et de la société - est primordiale. En empruntant la notion de culture aux anthropologues, le culturalisme cherche à rendre compte de l'intégration sociale des individus. En s'appuyant sur l'observation des sociétés archaïques, les culturalistes mettent en évidence l'influence prépondérante de la culture et des habitudes culturelles d'éducation sur la personnalité de base des individus.

Ce nouveau paradigme permet de dépasser les logiques d'instrumentalisation qui avaient présidé jusqu'ici au développement de la politique patrimoniale et culturelle. On le voit très nettement dans les cinq principes déterminés par la nouvelle politique de la culture telle qu'elle apparaît dans le Plan décennal des années 1990 :

la culture, un cadre pour le bien-être ;

la culture, un cadre créateur d'harmonie ;

la culture, un cadre de réflexion sur la paix ;

la culture, un cadre d'ouverture ;

la culture, un cadre de promotion pour la réunification du Sud et du Nord.

Il y donc clairement une volonté d'internationalisation de la culture coréenne à partir de sa culture traditionnelle. On sort d'une logique « obsidionale » et agonistique. À l'identitarisme nationaliste succède un idéal d'harmonie et de paix. Le patrimoine n'est plus seulement un instrument de résilience : il est désormais un facteur de reliance $»^{2}$. La politique de la Sixième République a inspiré les actions de promotion de la réunification du Sud et du Nord comme, par exemple, les travaux de

2. Le concept de « reliance » a été popularisé par le spécialiste des médias Marcell Bolle de Bal qui, pour bien distinguer la notion de celle de " connexion ", réintroduit les notions de sens et de finalité (voir son livre : 1985, La tentation communautaire. Les paradoxes de la reliance et de la contre-culture. Éditions de l'Université de Bruxelles, Bruxelles). 
standardisation de la langue coréenne, la recherche des données de base pour la culture unie et un travail d'ouverture de la culture au Nord.

Pour réaliser ces objectifs politiques, les gouvernements lancent des études scientifiques systématiques en créant différents instituts de recherche dédiés au patrimoine culturel tels que, par exemple, l'Institut de recherche sur le développement de la culture et les établissements de formation à la gestion de la culture et de la technique et de la conservation du patrimoine culturel.

Sous la présidence de Kim Young-sam, premier président civil (19931998), et bien que celui-ci fût conservateur et aux prises avec la crise financière asiatique, la politique de promotion des activités culturelles immatérielles fut maintenue et renforcée, comme s'il s'agissait désormais d'une donnée sociétale incontournable et indépendante des aléas politiques. Citons : les travaux du palais Gyeongbokgung ${ }^{3}$; les subventions importantes allouées aux projets de valorisation des patrimoines immatériels ; la construction d'établissements de formation au patrimoine culturel ; la politique de conservation des archives du patrimoine immatériel ; l'élargissement de la notion de patrimoine immatériel ; l'encouragement au développement des trésors vivants ; la recherche du patrimoine dispersé à l'étranger ; la volonté de faire émerger un patrimoine des Coréens unis et la réalisation d'échanges culturels avec la Corée du Nord par étapes ; l'organisation de la fête commune entre Sud et Nord à l'occasion du 50 anniversaire de l'Indépendance de la péninsule coréenne; le soutien à la diffusion mondiale de la culture coréenne; le développement des « Maisons de la Corée » à l'étranger ; et la recherche sur le patrimoine de la Corée du Nord.

En 1998, Kim Dae-jung devient président. Longtemps chef de l'opposition et partisan de la transition démocratique, il obtient le Prix Nobel de la Paix pour sa politique de réconciliation des deux Corées. Roh Moo-hyun (1946-2009) lui succède à la présidence en 2003 et poursuit notamment sa «politique du rayon de soleil » vers le Nord. Ces deux

3. Gyeongbokgung (경복궁), qui signifie « palais du Bonheur resplendissant », est un palais royal situé au nord de Séoul en Corée du Sud. Édifié en 1394 puis reconstruit en 1867, il est le principal des cinq grands palais construits sous la dynastie Joseon. Il avait été presque entièrement détruit par le gouvernement japonais au début du XXe siècle. 80 \% des bâtiments ont été démantelés et un énorme bâtiment du gouvernement japonais a été construit en face du secteur principal du palais. Un effort pour rétablir pleinement le palais de Gyeongbokgung dans son ancienne gloire est en cours depuis 1990. Le projet de restauration par le gouvernement sud-coréen est prévu jusqu'en 2030. 
présidences adoptent la devise suivante en matière de politique culturelle : « la culture créatrice de bien-être ». Quatre objectifs sont poursuivis :

la recherche de bénéfices économiques par la création active de la culture ;

la création de liens entre la société technologique et le monde de la culture et du patrimoine ;

le rapprochement des deux Corées par la culture et le patrimoine ;

la consolidation de la mixité culturelle coréenne alliant tradition et globalisation.

Durant cette période, l'expertise que la Corée du Sud avait acquise dans le domaine du patrimoine immatériel a suscité l'intérêt de l'UNESCO qui en faisait à ce moment un nouvel axe de sa politique. Ce concept s'est diffusé dans le monde entier. C'est ainsi que l'année 1997 a été nommée Année du patrimoine et de l'héritage culturel. Au tournant du millénaire, la Corée du Sud poursuit sa politique d'ouverture à l'international en s'approchant de références internationales comme celles de l'UNESCO. En 2003, la Corée du Sud fut le onzième État membre de l'UNESCO à ratifier la Convention pour la sauvegarde du patrimoine culturel immatériel. En 2009, la Corée s'est engagée à mettre en œuvre le réseau international pour la protection du patrimoine culturel immatériel au Forum international d'Asie.

\section{De la K-pop à l'Université du Patrimoine}

À partir de 2008, le gouvernement lance un plan « d'économie créative » associant étroitement industrie et culture. Il encourage le processus de globalisation de la culture coréenne, mais une culture bien éloignée du patrimoine traditionnel : K-pop, K-film et K-Drama... La $\mathrm{K}$-pop désigne une forme moderne de musique pop sud-coréenne recouvrant notamment les genres dance-pop, pop ballade, électronique, rock, hiphop. Elle révolutionne l'industrie musicale en Corée du Sud. À partir des services de réseaux sociaux et de la plateforme de partage vidéo YouTube, la K-pop étend son audience et prolifère grâce aux services de partage sur Internet. Au milieu des années 2000, le marché de la musique K-pop double ses ventes. Durant la première moitié de 2012, elle rapporte un total de 3,4 milliards de dollars. Si certains la considèrent comme une sous-culture réservée aux adolescents et aux jeunes adultes, il est intéressant de noter qu'un des effets imprévus de la K-pop est la promotion de l'étude du coréen, 
en France notamment.

La dernière importante initiative patrimoniale institutionnelle est sans conteste la création d'une université dédiée au patrimoine culturel à Buyeo. Cette opération témoigne de la cohérence et de la continuité des politiques publiques dans le domaine du patrimoine culturel en Corée du Sud.

Le premier président civil de Corée (Kim Young-sam) avait pris conscience que certains objectifs de développements civils ne pouvaient être atteints faute de personnel suffisamment qualifié. Ce besoin était particulièrement criant dans le domaine de la gestion du patrimoine. La Corée est forte d'un ensemble d'universités nationales généralistes (en principe une par région) et d'universités spécialisées : une Académie militaire, une Académie de police et des universités de technologie dont KAIST. C'est sur ce modèle d'université spécialisée qu'on imagine la création d'une institution de formation à la conservation du patrimoine. En 1996 paraît le décret de création de l'Université nationale du Patrimoine culturel coréen. Elle ouvre ses portes en 2013. Elle compte actuellement 560 étudiants à bac +4 et 150 étudiants en master. Son campus est conçu comme un ensemble bâti de style traditionnel. Il est implanté à Buyeo, la dernière capitale du royaume de Baekje ( $\mathrm{VI}^{\mathrm{e}}-\mathrm{VII}{ }^{\mathrm{e}}$ siècle, sud-ouest de la péninsule). Le choix du site n'est pas anodin. Le royaume de Silla (sudest de la péninsule), qui était sorti vainqueur de la rivalité entre les trois royaumes (Baekje, Goguryeo, Silla), avait développé surtout ses anciennes terres. Les richesses archéologiques du royaume de Baekje sont donc bien préservées et les étudiants sont ainsi immergés dans des vestiges d'un passé d'avant le royaume de Silla unifié.

Cette université destinée à la formation de personnels d'excellence dans son domaine spécialisé comporte trois facultés : la première est dédiée au patrimoine matériel, la deuxième au patrimoine immatériel, la troisième à la dimension technoscientifique de la protection du patrimoine. Elle est à l'image des préoccupations majeures des gestionnaires du patrimoine culturel. Il est trop tôt pour savoir si, comme KAIST (depuis 1971) en technologie, elle aura un rayonnement international incontestable en conservation du patrimoine culturel. Ajoutons que cette structure spécialisée est en compétition avec plus d'une dizaine de départements intégrés dans six universités nationales généralistes. Cette création montre l'importance de l'attachement de la société coréenne à son patrimoine et la volonté d'en faire une politique de rassemblement et de distinction. Mais elle montre également un souci de ne pas abandonner le patrimoine au domaine du simple folklore passéiste. 


\section{Conclusion}

Le contexte historique difficile dans lequel la Corée a évolué au XX siècle (notamment l'occupation japonaise) a constitué une menace majeure pour le patrimoine coréen, patrimoine matériel mais surtout patrimoine immatériel (langue, traditions, techniques, coutumes, etc.). La guerre finie, le Japon vaincu et la Corée enfin libérée, un nouveau drame se produit, drame qui n'est pas terminé à ce jour : la division de la Corée. Dans ces conditions, le patrimoine coréen a joué deux fonctions : il a été un facteur de résilience identitaire mais aussi, face à la Corée du Nord, un vecteur d'affirmation géopolitique. Ce qui est étonnant, c'est la capacité des régimes politiques successifs (de la dictature à la démocratie), certes avec des densités variables et des priorités parfois différentes, de considérer avec constance le patrimoine comme un enjeu national de première importance et d'y consacrer des moyens importants. Ce qui est également significatif, c'est l'évolution de la perception et des modes d'instrumentalisation du patrimoine : d'abord regardé comme un moyen d'affirmation nationaliste, il est devenu l'emblème d'une politique de réconciliation des deux Corées et un facteur de rayonnement mondial de la culture coréenne, tout en conciliant du mieux possible mémoire et modernité, traditions et innovations. 


\section{Références}

Asian Economy, 2012, 6 décembre.

Chang Gyoo Kim, 2012, A Safeguarding System for Cultural Heritage in Korea: Focused on the Activities of Restoration, Transmission and Protection of Designated Cultural Properties. Seoul, Prepared by Korea National University of Cultural Heritage, Cultural Heritage Administration (CHA).

Chosun Daily, 1951, 12 décembre.

Lee, Jang-Yeol, 2006, Korean Intangible Cultural Property Policy. Seoul, Kwandong Publishing Co. Ltd.

Jaen, Kwan-young, 2004, Research for establishing basic Law of Culture. Seoul, Korea Culture and Tourism Policy Institute.

Jang, Noo-hyun, 2005, « Research on a new direction of digitalization of cultural heritage ». Humanities Contents Issue 5: p.13-30.

Kim, Hyae-Jaeng, 2012, Cultural Policy Plan for Promoting Cultural Diversity. Seoul, Korea Culture Tourism Institute.

Kim, Sae-hun, 2012, Cooperation between Culture and Art Policy and other policy Areas. Seoul, Korea Culture Tourism Institute.

République de Corée, 2007, Loi sur la protection du patrimoine culturel. (Loi n 961 du 10 janvier 1962, amendée par la Loi no 8346 du 11 avril 2007). , 1962, 2011, 2017, Loi sur la protection culturelle.

$-, 1962,2011,2017$, Loi sur la protection culturelle. immatériels (Loi n¹3248, 27 mars).

— ligne, http://www.mogaha.go.kr/

— s.d., Biens culturels nommés par l'État, en ligne, http://www.index. go.kr/potal/main/EachDtlPageDetail.do?idx_cd=2750

Rozin, Philippe, 2006, "Le concept de culturalisme dans les sciences anthropologiques, de Tylor à Lowie ». Le Philosophoire 27 (2) : 151-176.

Soul, Ho-jeong, 2012, « Les défenseurs discrets des trésors humains vivants. Le patrimoine culturel immatériel au $\mathrm{XX}^{\mathrm{e}}$ siècle ». Koreana 13 (3) : 16-19.

Yim, H. S., 2002, "Cultural identity and cultural policy in South Korea », The International Journal of Cultural Policy 8 (1): 37-48. 